\title{
Effect of Particulate Reinforcements on the Mechanical Properties of Al6061-WC and Al6061-Gr MMCs
}

\author{
A. R. K. Swamy', A. Ramesha ${ }^{2}$, G.B. Veeresh Kumar ${ }^{3 *}$ J. N. Prakash ${ }^{4}$ \\ ${ }^{1}$ Research Scholar, Dr. M.G.R Educational \& Research Institute, Chennai \& Department \\ Mechatronics Engineering, Acharya Institute of Technology, Bangalore, India. \\ ${ }^{2}$ GATES Institute of Technology, Gooty, Andhra Pradesh, India. \\ ${ }^{3}$ Department of Mechanical Engineering, Amrita Vishwa Vidyapeetham, Bangalore, India. \\ ${ }^{4}$ Department of Mechanical Engineering, Alpha College of Engineering, Bangalore, India. \\ *Corresponding author: veeru232@yahoo.com
}

\begin{abstract}
This paper presents the comparative study of the mechanical properties of Al6061-Tungsten carbide composites containing Tungsten carbide (WC) particulate, and Al6061-graphite particulate composites containing graphite particles. The reinforcing particulates in the MMCs vary from $0 \%$ to $4 \%$ by weight. The 'vortex method' of production was employed to fabricate the composites, in which the reinforcements were poured into the vortex created by stirring the molten metal by means of a mechanical agitator. The composites so produced were subjected to a series of tests.
\end{abstract}

The results of this study revealed that as the Tungsten carbide particle content was increased, there were significant increases in the ultimate tensile strength, hardness and Young's modulus, accompanied by a reduction in its ductility. There was, however, only a very marginal increase in the compressive strength, where as in graphite reinforced composites as the graphite content was increased, there were significant reduction in hardness and monotonic increases in the ductility, ultimate tensile strength (UTS), compressive strength and Young's modulus of the composite, An attempt is made in the paper to provide explanations for these phenomena.

Keywords: Aluminum alloys, Tungsten carbide, Graphite, Composites, Mechanical properties.

\section{INTRODUCTION}

Metal matrix composites (MMCs) are increasingly becoming attractive materials for advanced aerospace applications because their properties can be tailored through the addition of selected reinforcements [1-2]. In particular, particulate reinforced MMCs have recently found special interest because of their specific strength and specific stiffness at room or elevated temperatures [3]. It is well known that the elastic properties of metal matrix composites are strongly influenced by micro structural parameters of the reinforcement such as shape, size, orientation, distribution and volume fraction [4]. 
Aluminium-based MMCs have received increasing attention in recent decades as engineering materials. The introduction of a ceramic material into a metal matrix produces a composite material that results in an attractive combination of physical and mechanical properties which cannot be obtained with monolithic alloys. There is an increasing need for knowledge about the processing techniques and mechanical behaviour of particulate MMCs in view of their rising production volumes and their wider commercial applications. Interest in particulate reinforced MMCs is mainly due to easy availability of particles and economic processing technique adopted for producing the particulate-reinforced MMCs. Aluminium alloy-based particulate-reinforced composites have a large potential for a number of engineering applications. Interest in reinforcing $\mathrm{Al}$ alloy matrices with ceramic particles is mainly due to the low density, low coefficient of thermal expansion and high strength of the reinforcements and also due to their wide availability. Among the various useful aluminium alloys, aluminium alloy 6061 is typically characterized by properties such as fluidity, castability, corrosion resistance and high strength-weight ratio. This alloy has been commonly used as a base metal for MMCs reinforced with a variety of fibres, particles and whiskers [5-7].

In recent years, considerable work has been done on graphite reinforced metal matrix composites which exhibit low friction, low wear rate and excellent antiseizing properties. The graphite in these composites presumably imparts improved tribological properties to the composites through the formation of a graphite-rich film on the tribo-surface which provides solid lubrication. Journal bearings made of graphite particle dispersed composites perform much better than conventional bearing alloys [8]. Graphite particles of size ranging from 50 to $200 \mu \mathrm{m}$ yield the best results [9]. Manufacturing automotive pistons out of graphite reinforced composites instead of other conventional materials resulted in a saving of $5-7 \%$ on fuel and lubricating oil [10]. The presence of graphite in the matrix improves its oil spreadability over the contact surface, thus reducing the tendency to score or seize. Graphite, which consists of carbon atoms arranged in a layer-like structure, displays a very low coefficient of friction while sliding on another clean surface, thus suggesting that it can be used as solid lubricants [11]. Because of this solid lubricative property, graphite in the form of particles has a wide range of applications in composite materials which are used to make components requiring great wear resistance such as engine bearings, pistons, piston rings and cylinder liners [12]. Although fibre reinforcements lead to marked enhancement in its properties, composites using particulate or discontinuous reinforcement for high-volume applications are being increasingly sought. The most widely used reinforcements have been $\mathrm{SiC}$, alumina, graphite, $\mathrm{B}_{4} \mathrm{C}$ and $\mathrm{TiC}$. These have been used to achieve improvements in selected properties. Most of the published data pertain to the mechanical properties of particulate-reinforced MMCs deal with tensile properties while only a relatively small amount of data has been obtained dealing with compression properties, although it is generally known that the compressive strength of an MMC is invariably higher than its UTS. Such MMCs are quite brittle compared with monolithic materials and have values of percentage elongation typically less than 5 per cent. For unreinforced aluminium alloy 6061, Awerbuch et al. [13] have shown that the deformation in compression could be greater than 50 per cent for specimens having a length-diameter ratio L/D equal to unity. Such effects could also be responsible for the apparently high value of the compressive strength. Hence, in the present investigation, importance is also given to the compressive properties of the MMCs, together with the tensile properties such as the UTS, ductility, hardness and Young's modulus.

In the present investigation, aluminium alloy 6061 was used as the matrix material. Al6061 alloy has the highest strength and ductility of the aluminium alloys with excellent 
machinability and good bearing and wear properties [14]. Most of the particulate reinforced metal matrix composites are produced by liquid metallurgy, sometimes known as the 'vortex method' [15], although many different processes for fabricating these cast composites are also available which have been reported by various researchers. In the present work, the 'vortex method' of producing AMC's, in which Tungsten carbide and graphite particulates have been used as the candidate reinforcements of particulate sizes ranging from 5 to $15 \mu \mathrm{m}$ and added to the vortex formed in the A16061 melt above its liquidus temperature. Since the hardness, ultimate tensile strength (UTS), compressive strength, Young's modulus and ductility of the composite material are all vital properties of a structural material, the present investigation aims at studying these properties in the Al6061 alloy-particulate composites.

\section{EXPERIMENTAL DETAILS}

The properties of materials adopted and methods followed for the fabrication and testing of MMCs in the present studies are presented in the following sections.

\subsection{Matrix and Reinforcement Materials Details}

The matrix for the present studies selected was Al6061 alloy and were procured from Fenfee Metallurgicals, Bangalore, in the form of ingots. The chemical composition of Al6061 alloy is given in Table 1. The reinforcing materials selected were tungsten carbide (WC) of $5 \mu \mathrm{m}$ size and graphite $(\mathrm{Gr})$ of $15 \mu \mathrm{m}$ particle size and the properties of the matrix and reinforcement materials used are presented in the Table 2.

Table 1. Chemical Composition of Al6061 by Weight percentage.

\begin{tabular}{|c|c|c|c|c|c|c|c|c|c|}
\hline Chemical Composition & Si & Fe & Cu & Mn & Mg & $\mathbf{C r}$ & $\mathbf{Z n}$ & $\mathbf{T i}$ & $\mathbf{A l}$ \\
\hline Al6061 & 0.62 & 0.23 & 0.22 & 0.03 & 0.84 & 0.22 & 0.10 & 0.1 & Bal \\
\hline
\end{tabular}

Table 2. Properties of matrix and reinforcement materials.

\begin{tabular}{|c|c|c|c|c|}
\hline Material & $\begin{array}{c}\text { Elastic Modulus } \\
(\mathbf{G P a})\end{array}$ & $\begin{array}{c}\text { Density } \\
(\mathbf{g} / \mathbf{c c})\end{array}$ & $\begin{array}{c}\text { Hardness } \\
(\mathbf{H B 5 0 0})\end{array}$ & $\begin{array}{c}\text { Tensile Strength } \\
(\mathbf{M P a})\end{array}$ \\
\hline $\mathrm{Al6061}$ & $70-80$ & 2.7 & 30 & 115 \\
\hline $\mathrm{WC}$ & 627 & 14.9 & $1630^{* *}$ & 5000 \\
\hline $\mathrm{Gr}$ & $8-15$ & 2.09 & $1.7^{*}$ & $20-200$ \\
\hline
\end{tabular}

*Mohs scale; $* * \mathrm{Kg} / \mathrm{mm}^{2}$

\subsection{Preparation of Composites}

The Al6061-graphite composites were prepared by the vortex method [9]. The graphite contents used for the preparation of the composites were $0 \%, 1 \%, 2 \%$ and $4 \%$. This is because graphite compositions of $7 \%$ and above would lead to rejection from the melt [16]. Addition of graphite into the molten aluminium alloy melt above its liquidus temperature of $500{ }^{\circ} \mathrm{C}$ was carried out by creating a vortex in the melt using a mechanical stainless steel stirrer coated with aluminite (to prevent migration of ferrous ions from the stirrer material into the aluminium alloy melt). The melt was rotated at a speed of $500 \mathrm{rpm}$ in order to create the necessary vortex. The graphite particles were preheated to $400{ }^{\circ} \mathrm{C}$ and added to the melt through the vortex. The graphite particles were added, and the melt was thoroughly stirred and subsequently degassed by passing hexachloroethane $\left(\mathrm{C}_{2} \mathrm{Cl}_{6}\right)$ solid degasser. The molten metal was then poured into permanent moulds for casting. The above vortex method was 
followed to prepare Al6061-Tungsten Carbide composites; the size of WC was $5 \mu \mathrm{m}$. The preheated particles were introduced into the vortex and stirred for eight minutes at a speed of $500 \mathrm{rpm}$. A pouring temperature of $720{ }^{\circ} \mathrm{C}$ was adopted and the molten composite was poured into preheated cast iron moulds. The extents of incorporation of particles into the matrix alloy were varied from 1 to $4 \mathrm{wt} \%$ in the steps of $1 \mathrm{wt} \%$ increment. The after effective degassing the molten metal was then poured into permanent moulds for casting. The cylinders of 22 X $210 \mathrm{~mm}^{2}$ cast MMCs of Al6061-Gr and Al6061-WC were obtained.

\subsection{Testing of Specimens}

Carefully polished and mirror finished specimens were examined under NIKHON - Japan make, ECLIPSE 150 model up right metallurgical microscope to obtain microphotographs. Tensile tests were conducted at room temperature using a universal testing machine (UTM) in accordance with ASTM Standard E 8-82. The tensile specimens of diameter $12.5 \mathrm{~mm}$ and gauge length $60 \mathrm{~mm}$ were machined from the cast composites with the gauge length of the specimens parallel to the longitudinal axis of the castings.

For each composite, four tensile test specimens were tested and the average values of the UTS, Young's modulus and ductility were measured. The hardness tests were conducted in accordance with ASTM Standard E 10 using a Brinell hardness tester with a ball indenter of $10 \mathrm{~mm}$ diameter and a load of $500 \mathrm{~kg}$. The load was applied for $30 \mathrm{sec}$. Eight hardness readings were taken for each specimen at different locations to circumvent the possible effects of particle segregation. Compression tests were conducted on a UTM in accordance with ASTM Standard E 9 at room temperature. In this test the compression loads were gradually increased and the corresponding strain was measured until the specimen failed. Each result is an average of four readings.

\section{RESULTS AND DISCUSSIONS}

\subsection{Microstructure Studies}

Figures 1 and 2 show the optical micrographs of Al6061 alloy, Al6061-Gr and A16061-WC composites. Micrographs reveal that there is fairly uniform distribution of WC/Gr particulates throughout the matrix alloy and the porosity is lower. It is reported that higher hardness is always associated with lower porosity of the MMCs [17]. Also, it can be observed that there is good bonding between the matrix and the reinforcement particulates resulting in better load transfer from the matrix to reinforcement material.

\subsection{Hardness}

Hardness, is the measure of a material's resistance to surface indentation, also it is a function of the stress required to produce some specific types of surface deformation. Figure 3 is a graph showing the effect of graphite and WC reinforcements on the hardness of cast Al6061graphite and A16061-WC particulate composites. Each value represented is an average of six measurements. The results are repeatable in the sense that each individual result did not vary more than $5 \%$ from the mean value. 


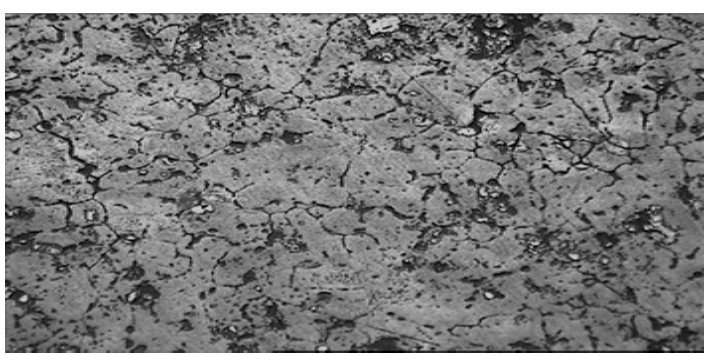

a) Al6061 alloy

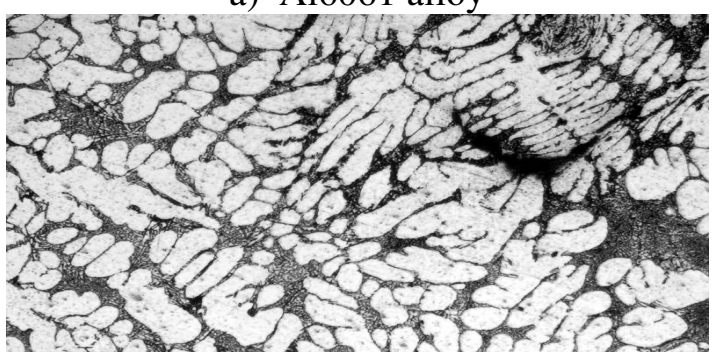

b) Al6061-1 wt\% Graphite

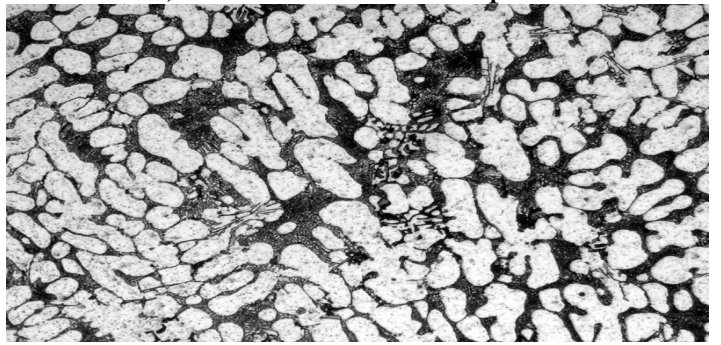

c) Al6061-2 wt\% Graphite

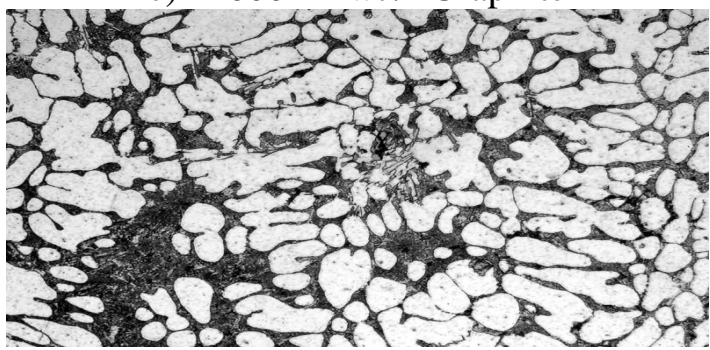

d) Al6061-3 wt\% Graphite

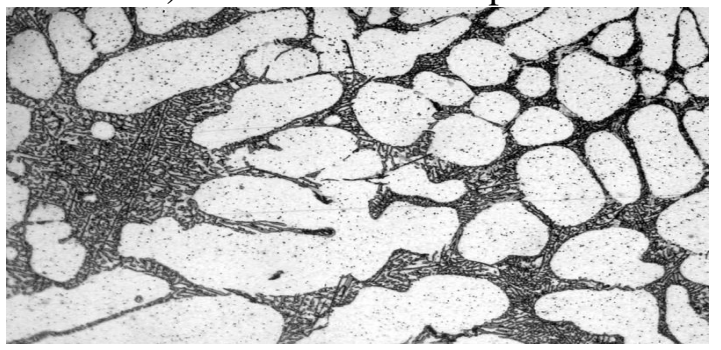

e) Al6061-4 wt\% Graphite

Figure 1. Micrographs of Al6061 and Al6061-Gr MMCs at $100 \mathrm{X}$ magnification for $50 \mu \mathrm{m}$.

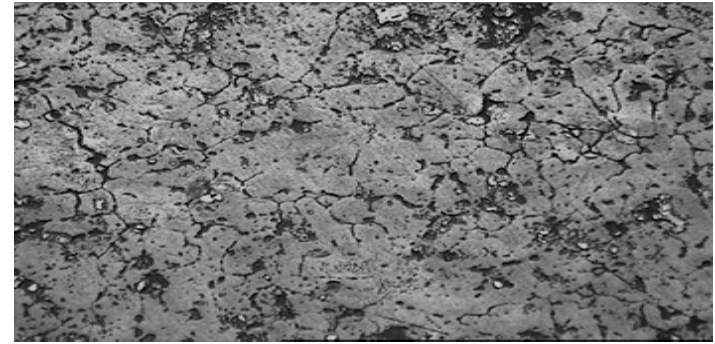

a) Al6061 alloy
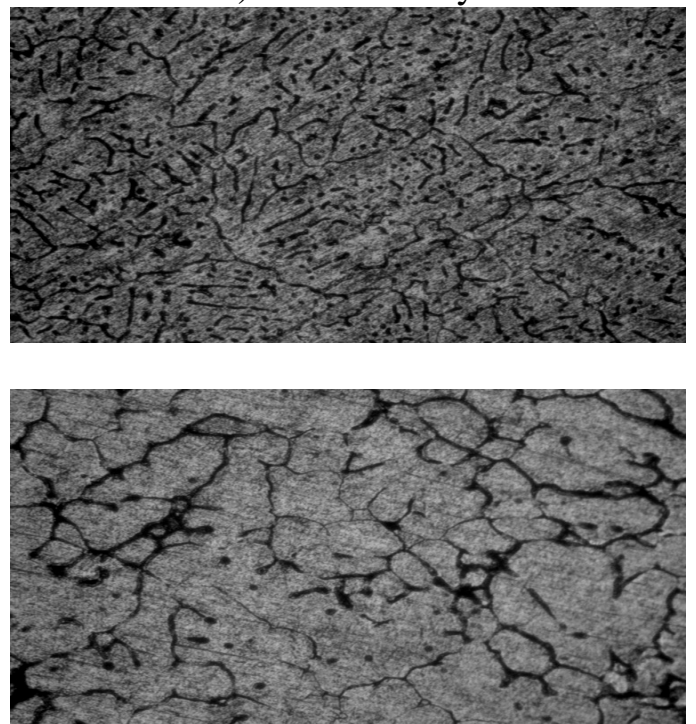

c) Al6061-2 wt\% Tungsten Carbide

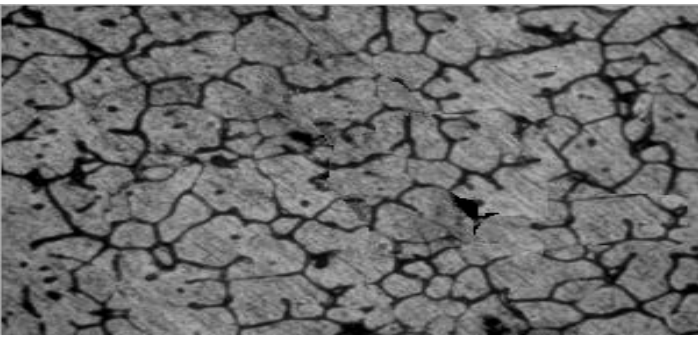

d) Al6061-3 wt\% Tungsten Carbide

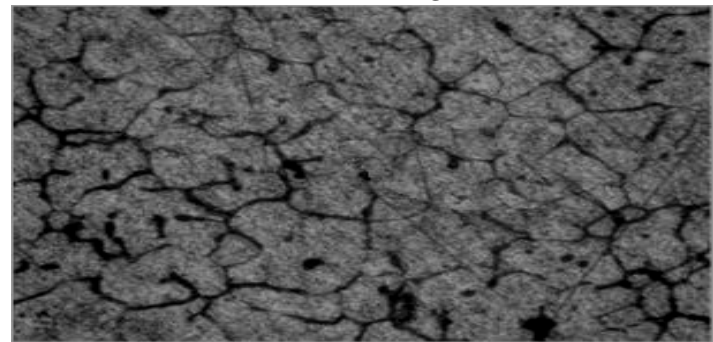

e) Al6061-4 wt\% Tungsten Carbide

Figure 2. Micrographs of Al6061 and Al6061-WC MMCs at $100 \mathrm{X}$ magnification for $50 \mu \mathrm{m}$. 


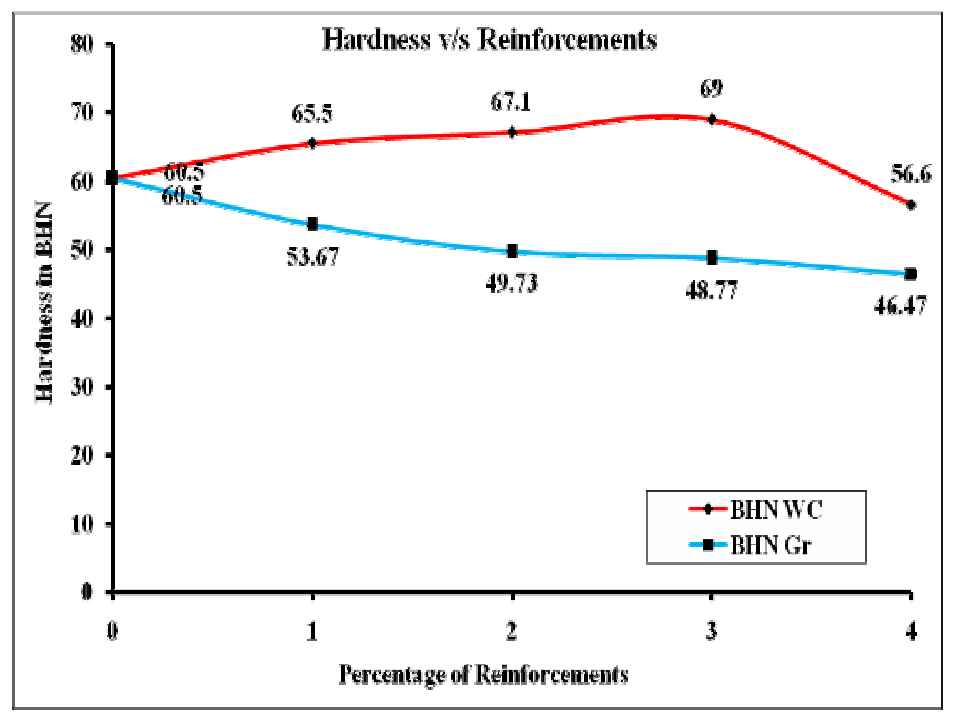

Figure 3. Effect of the WC and graphite content on Hardness.

It can be seen that as the graphite content increases, the hardness of the composite material decreases monotonically by significant amounts. In fact, as the graphite content is increased from $0 \%$ to $4 \%$ the hardness decreases by about $23 \%$. In case of metals, as the Ultimate Tensile Strength (UTS) of a material increases, so would the hardness but the opposite is seen in this particular composite, whereby the hardness drops as the UTS increases [18]. There is a good reason for this phenomenon, though, since graphite, being a soft dispersoid, does not contribute positively to the hardness of the composite. K.H.W Seah et al., [19] have reported a reduction in hardness from $107 \mathrm{BHN}$ to $77 \mathrm{BHN}$ (about $28 \%$ differences) on addition of similar weight percentages of graphite to ZA-27 (Zinc Aluminium) alloy. Such a monotonic decrease in the hardness of the composite as graphite content is increased poses a limit to how much graphite may be added to enhance its other mechanical properties, since hardness is directly related to wear resistance, a compromise is necessary when deciding how much graphite should be added to enhance the ductility, UTS, compressive strength, and Young's modulus of the composite without sacrificing too much of its hardness.

Further the hardness of the Al6061-WC particulate composite is increasing with the increase of reinforcing particulate content. The hardness value of the composite is higher than that of its matrix alloy. It was observed that the hardness value improved up to $3 \mathrm{wt} \%$ of WC, the hardness increased by about $14 \%$ and again the hardness is decreased for 4 wt. \% of WC. Better hardness was found at $3 \mathrm{wt}$. \% of WC. Hence from the studies it can be concluded that up to $3 \mathrm{wt}$. \% of WC can be successfully incorporated into the matrix.

\subsection{Ultimate Tensile Strength}

Figure 4 is a graph showing the effect of reinforcement content on the Ultimate Tensile Strength (UTS) of cast A16061-graphite and A16061-WC particulate composites. Each value represented is an average of six measurements. The results are repeatable in the sense that each individual result did not vary more than $5 \%$ from the mean value. It can be seen that as the graphite content increases, the UTS of the composite material increases monotonically by significant amounts. In fact, as the graphite content is increased from $0 \%$ to $4 \%$, the UTS increases by about $38 \%$. These results are in accordance with those obtained by Pillai [20] 
who reported similar findings. This increase in UTS may be due to the graphite particulates acting as barriers to dislocations in the microstructure [21].

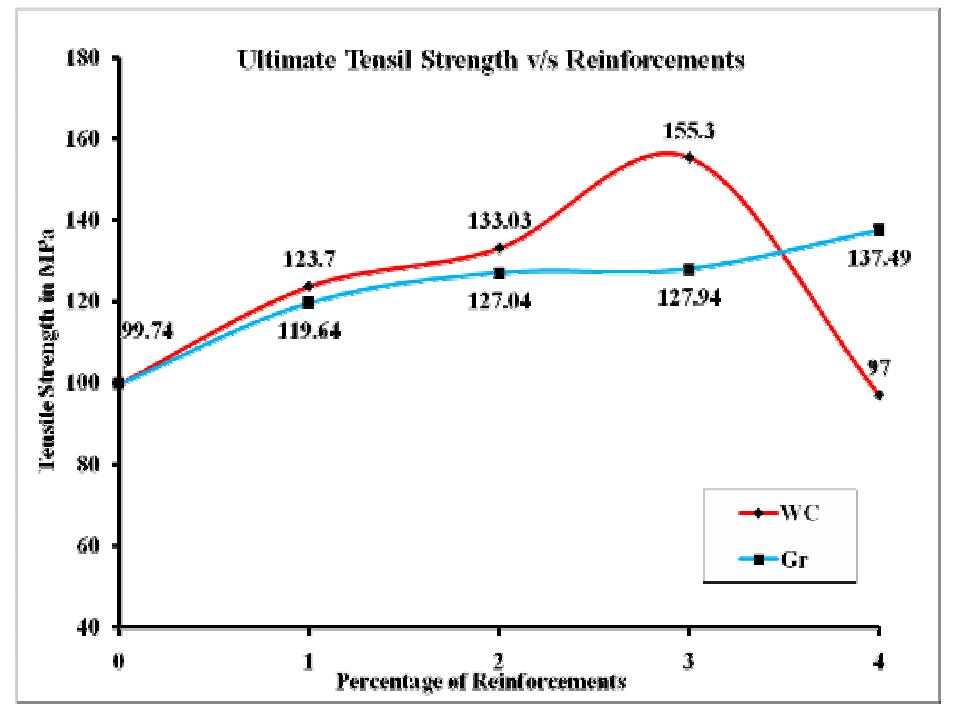

Figure 4. Effect of the WC and graphite on Ultimate Tensile Strength.

It was observed that as the wt. $\%$ of WC is increased from $0 \%$ to $4 \%$ it was observed that the tensile strength value improved up to $3 \mathrm{wt}$. \% WC and the tensile strength decreased for $4 \mathrm{wt}$. $\% \mathrm{WC}$. Better tensile strength was found at $3 \mathrm{wt} \% \mathrm{WC}$. As the WC content is increased from $0 \%$ to $3 \%$, the UTS increases by about 55\%. The decrease in tensile strength of the composites beyond $3 \mathrm{wt}$. \% WC is attributed to improper bonding between the matrix and reinforcement materials. Finer the grain size better is the hardness and strength of composites leading to lowering of wear rates. The increase in UTS can be attributed to the presence of hard WC particulates that impart strength to the matrix alloy, thereby providing enhanced resistance to tensile stresses. There is a reduction in the interspatial distance between the hard WC particulates, which causes an increase in the dislocation pile-up as the particulate content is increased. This leads to a restriction in the plastic flow due to the random distribution of the particulates in the matrix, thereby providing enhanced tensile strength to the composites.

Similar observations were made by Ghosh and Ray [22], who fabricated $\mathrm{Al}_{2} \mathrm{O}_{3}$ particulatereinforced aluminium alloy composites using the compo-casting method and by McCoy et al. [23] who produced $\mathrm{TiB}_{2}$-particulate-reinforced aluminium alloy composites with particulate contents ranging from 10 to $25 \mathrm{vol} \%$. An increase in the UTS was observed as the $\mathrm{TiB}_{2}$ content was increased from 10 to $18 \mathrm{vol} \%$.

\subsection{YOUNGS MODULUS}

Figure 5 is a graph showing the percentage variation in Young's modulus provides a comparative insight in to the actual experimental results recorded for the Al6061-WC and Al6061-graphite particulate reinforced composites. The effect of graphite content on the Young's modulus of cast Al6061-graphite particulate composites. As in the cases of UTS described above, it can be seen that as the graphite content increases, the Young's modulus of the composite material increases monotonically by significant amounts. In fact, as the graphite content is increased from $0 \%$ to $4 \%$, the Young's modulus increases by about $9 \%$. Similar results have been obtained in aluminium matrix composites where the Young's 
modulus has been reported to increase with increase in the content of the reinforcing material, regardless of the type of reinforcement used [24]. Nevertheless, it must be emphasized at this point that the values of Young's modulus presented in this paper are only useful for the purpose of comparison among themselves.

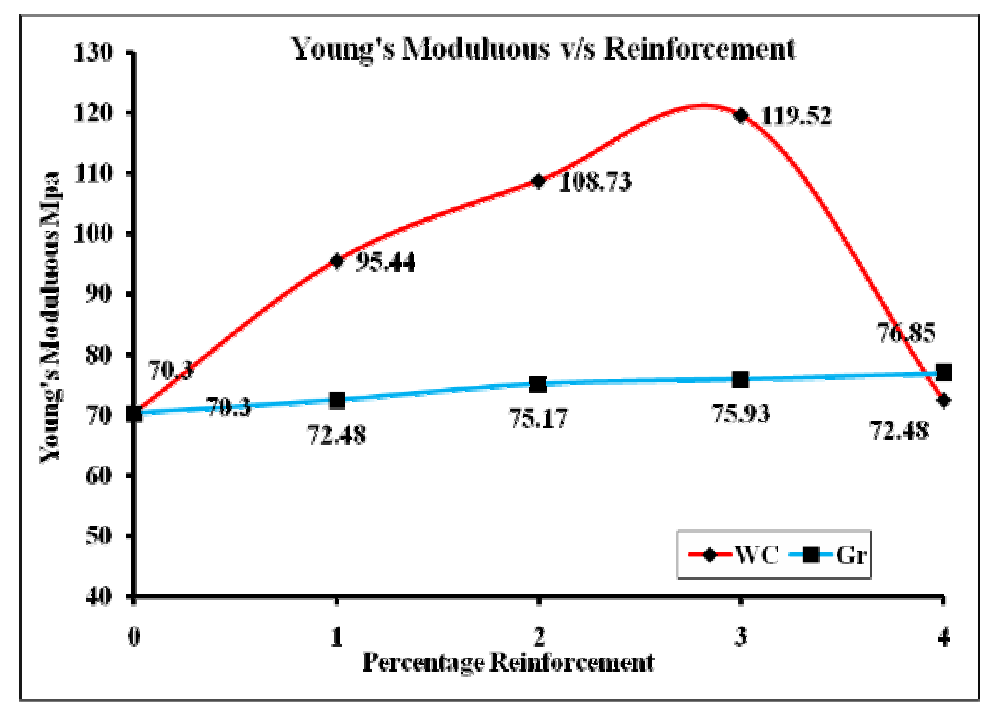

Figure 5. Effect of the WC and graphite content on Young's Modulus.

It can be seen that as the WC content increases, the Young's modulus of the composite material increases monotonically by significant amounts. In fact, as the WC content is increased from $0 \%$ to $3 \%$, the Young's modulus increases by about $70 \%$ and when increased from 3\% to 4\% the Young's modulus decreases monotonically as shown in the Figure 5. This increase in Young's modulus is broadly in line with the rule-of-mixtures prediction. McDanels [24] who obtained similar results for particle-reinforced aluminium composites reported that Young's modulus increases with increase in reinforcement content regardless of the type of reinforcement used.

\subsection{Ductility}

Figure 6 is a graph showing the reinforcement content on the ductility of cast Al6061graphite and Al6061-WC particulate composites (measured in terms of percentage elongation). As the graphite content increases, the ductility of the composite material increases monotonically by significant amounts, i.e., when the graphite content is increased from $0 \%$ to $4 \%$ the ductility was found to increases by about $165 \%$. The effect of graphite is expected to be mechanical in nature since the particles are unreactive with the matrix phase [20]. This considerable increase in ductility is due to the graphite additions, being an effective solid lubricant [25, 8-11], eases the movement of grains along the slip planes. 


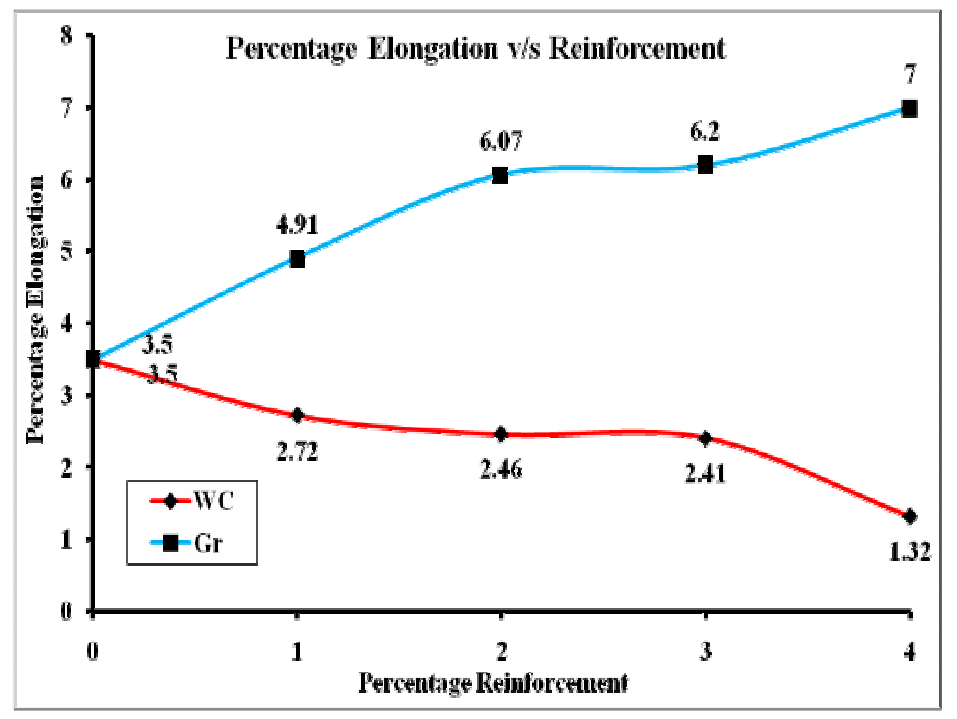

Figure 6. Effect of the WC and graphite content on Ductility

The elongation of the composite material A16061-WC is less as compared to that of the cast Al6061 alloy. It is clear that the composite material exhibits higher brittleness with increased filler content (WC), in other words the matrix material suffers with ductility due to the influence of the reinforcement material. In fact, as the WC content is increased from $0 \%$ to $4 \%$ the ductility drops by about $100 \%$. These results tally with those obtained by other researchers $[26,27]$ who also observed that the ductility of the composites decreases with increase in the reinforcement content. This decrease in ductility in comparison with the matrix alloy is a most commonly encountered disadvantage in discontinuously reinforced MMCs [28]. The reduction in ductility can be attributed to the presence of a hard ceramic phase that is prone to localized crack initiation and increased embrittlement effect due to local stress concentration sites at the reinforcement-matrix interface. Hence, the introduction of this hard secondary ceramic phase creates slip regions. Moreover, the reinforcing particulates resist the passage of dislocations either by creating stress fields in the matrix or by inducing large differences in the elastic behaviour between the matrix and the dispersoid. Mummery et al. [28] are of the opinion that this loss in ductility is probably due to the voids which nucleate during the plastic straining of the reinforcement.

\subsection{Compressive Strength}

Figure 7 is a graph showing the effect of graphite content on the compressive strength of cast Al6061-graphite particulate composites. It can be seen that as the graphite content increases, the compressive strength of the composite material increases monotonically by significant amounts. In fact, as the graphite content is increased from $0 \%$ to $4 \%$, the compressive strength increases by about $56 \%$ and this increase in compressive strength may be due to the graphite particles acting as barriers to dislocations in the microstructure. 


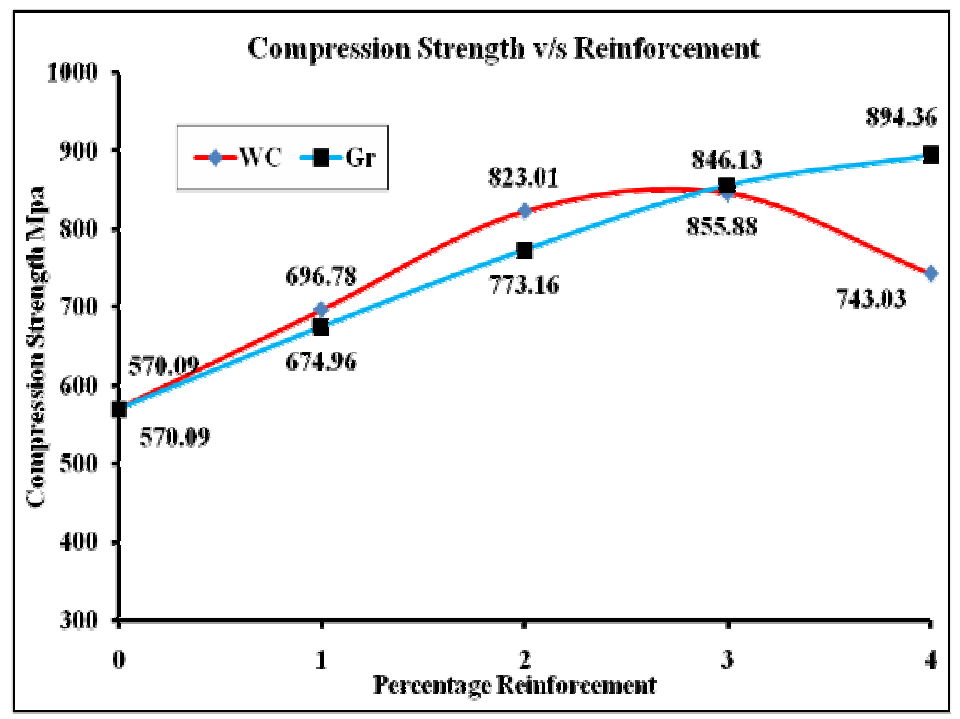

Figure 7. Effect of the WC and graphite content on compression strength.

Figure 7 is a graph showing the effect of the WC particulate content on the compressive strength of the composites. From Figure 7 it can be observed that the compressive strength of the composites are higher than that of the base alloy because the WC is having very high compressive strength then the matrix material and further it can be observed that with increase in WC content the composites compressive strength also increases. As the wt $\%$ of $\mathrm{WC}$ is increased from $1 \%$ to $4 \mathrm{wt} \%$, the compressive strength value improved up to $3 \mathrm{wt} \%$ WC, the compressive strength increased by about $48 \%$ and the compressive strength decreased for $4 \mathrm{wt} \% \mathrm{WC}$. Better compressive strength was found at $3 \mathrm{wt} \% \mathrm{WC}$.

Similar results were observed by earlier researchers such as Webster [29] and Awerbuch et al. [13] when they conducted tests on whisker-reinforced composites. Towle and Fried [30] compared the compressive and tensile properties of magnesium-based MMCs and observed similar trends. The increase in compressive strength is chiefly due to the decrease in the interparticle spacing between the WC particles, since WC is much harder than aluminium alloy 6061. The presence of the WC particles resists deforming stresses, thus enhancing the compressive strength of the composite material. The increase in compressive strength is not as spectacular as those seen in the UTS, hardness and Young's modulus because the compressive strength of the unreinforced matrix material itself is already very high, in fact several times the UTS. There is only a very marginal increase in the compressive strength when a secondary ceramic phase is introduced. Nevertheless, the addition of hard ceramic particulates has caused the MMCs to behave as brittle rather than ductile materials, as is evident from the above results.

\section{CONCLUSIONS}

The significant conclusions of the studies on Al6061-WC and Al6061-Gr metal matrix composites are as follows.

1. Al6061-WC and Al6061-graphite composites were prepared successfully using liquid metallurgy techniques by incorporating the reinforcing particulates up to $4 \mathrm{wt} \%$. 
2. It was found that increasing the graphite content within the aluminum matrix results in significant increases in the ductility, UTS, compressive strength and Young's modulus, but a decrease in the hardness.

3. A compromise is necessary when deciding how much graphite should be added to enhance the mechanical properties of the composite without sacrificing too much of its hardness and hence its wear resistance.

4. The properties of the cast A16061-WC composites are significantly improved by varying the amount of WC. It was found that increasing the WC content within the matrix material, resulted in significant improvement in mechanical properties like hardness, tensile strength, and compressive strength at the cost of reduced ductility.

5. Highest values of mechanical properties like hardness, tensile strength and compressive strength were found at $3 \mathrm{wt} \% \mathrm{WC}$.

\section{ACKNOWLEDGEMENTS}

The authors express their thanks to Shri B. M. Reddy, President, Mr. B. Premnath Reddy, Chairman, Dr. G. P. Prabukumar, principal, Dr. K. G. Satyanarayan, Research Director, Acharya Institute of Technology, JMJ Education Society, Bangalore, Karnataka, India for the support and encouragement during this research studies.

\section{REFERENCES}

[1]. Everett R.K and Arsenault R.J. "Metal Matrix Composites: Mechanisms and Properties", 1991 (Academic Press, San Diego).

[2]. Kocjak M.J., Kahtri S.C., Allison J.E and Jones J.W., "Fundamentals of Metal Matrix Composites" (Ed S.Suresh, A.Mortensen and A.Needleman, 1993 (ButterworthHeinemann, Boston)).

[3]. Lloyd D.J. and Brotzen F.R., "Particle reinforced aluminium and $\mathrm{Mg}$ matrix composites" Int. Mater. Rev; 1994, 39,1-39.

[4]. Lei M. and Ledbetter H., "Communications: elastic constants of $\mathrm{SiC}_{\mathrm{p}} / \mathrm{Al}$ : measurements and modeling”, Metall. Mater. Trans., 1994, 25A, 2832-2835.

[5]. Vogelsang M., Arsenault R. J. and Fisher R. M., "In-situ HVEM study of dislocation generation at $\mathrm{Al} / \mathrm{SiC}$ interfaces", Metall. Trans. A, 1986, 17, 379.

[6]. Pai B. C., Ray S., Prabhakar, K. V. and Rohatgi P. K., "Fabrication of aluminiumalumina (magnesia) particulate composites in foundries using magnesium additions to the melts", Mater. Sci. Engng, 1976,24,31.

[7]. Sato A. and Mehrabian R., "Aluminium matrix composites: fabrication and properties", Metall. Trans. B, 1976, 7, 443.

[8]. Biswas S., Santharam A., Rao N.A.P Narayanaswamy, K. Rohatgi, P. and Biswas S. K. Tribology International 1980, 8, 171.

[9]. Pai B. C., Pillai R.M. and Sathyanarayana K.G, "Prospects for graphite aluminium composites in engineering industries" Indian Journal of Engineering and Materials Science 1994, 1, October, 279.

[10]. Krishnan B.P., Raman N., Narayanaswamy K and Rohtagi P.K. Wear, 1980, 60, 1.

[11]. Bragg W. L., "Introduction to Crystal Analysis", Bell and Son London, 1928, p. 64. 
[12]. Pillai U. T. S., Pandey R. K. and Nagam K. D. P., "Deformation and fracture of aluminium graphite and aluminium zircon particulate composites" Proc of the $5^{\text {th }}$ International Conference on Composite Materials. TMS Publications, 1985, p. 895.

[13]. Awerbuch J., Goering J. and Busking K., "In Mini Mechanics Analysis and Testing of Short Fibre Composites: Experimental Methods and Results", Vol. 121, 1988. pp964 (American Society for Testing and Materials, Philadelphia. Pennsylvania).

[14]. Smith W. F, "Structure and Properties of Engineering Alloys", $2^{\text {nd }}$ edn, McGraw-Hill, New York, 1993, p. 566.

[15]. Badia F. A., McDonald D. F. and Pearson J. R. Trans. AFS 1971, 79, 269.

[16]. Biswas S., Dwarakadasa E. and Biswas S. K, "Bearings and wear properties of cast graphite aluminium composites", Proc. of All India Seminar on Aluminium, 1979.

[17]. C.S. Ramesh, R. Keshavamurthy, B.H. Channabasappa, Abrar Ahmed, "Microstructure and mechanical properties of $\mathrm{Ni}-\mathrm{P}$ coated $\mathrm{Si}_{3} \mathrm{~N}_{4}$ reinforced Al6061 composites" Materials Science and Engineering A 502 (2008) 99-106.

[18]. A. Ramesh, J. N. Prakash, A. S. Shiva Shankare Gowda and Sonnappa Appaiah "Comparison of the Mechanical Properties of AL6061/Albite and AL6061/Graphite Metal Matrix Composites" Journal of Minerals \& Materials Characterization \& Engineering, Vol. 8, No.2, pp 93-106, 2009.

[19]. K.H.W Seah, S.C.Sharma, B.M.Girish, "Mechanical properties of cast ZA-27/graphite particulate composites", Materials and Design, 1995,16, 271- 275.

[20]. Pillai U. T. S and Pandey R. K, "Studies on mechanical behaviour of cast and forged aluminium graphite particle composites”, Journal of Composite Materials 1989, 23, February, 108.

[21]. Dieter G. E, "Mechanical Metallurgy", SI metric edition, McGrawHill, New York, 1988, pp. 212-19.

[22]. Ghosh P. K. and Ray S. J. Mater. Sci., 1986, 21, 1167.

[23]. McCoy J. M., Prones C. and Warner F. E. 1. Mater. Sci., 1988, 1, 37.

[24]. McDanels D. L., "Analysis of stress-strain, fracture and ductility behaviour of aluminium matrix composites containing discontinuous SiC reinforcement", Metall. Trans. A, 1985, 16, 1105-1115.

[25]. Rohatgi P. K., Ray S. and Lin Y, “Tribological properties of metal matrix graphite particle composites", International Materials Review, 1992, 37, No.3, 129.

[26]. Beitz W. and Kuttner K. H. (Eds) Dubbel Handbook of Mechanical Engineering, 1994, p. D8 (Springer-Verlag, London).

[27]. Zhu H. X. and Liu S. K, "Mechanical properties of squeeze cast zinc alloy matrix composites containing alpha-alumina fibers" Composites, 1993, 24(6), 437.

[28]. Mummery P. M., Derby B. and Scruby C. B, "Acoustic emission from particulate reinforced metal matrix composites", Acta Metall., 1993,41,1431.

[29]. Webster D. Metall. Trans. A, 1982, 13A, 511.

[30]. Towle D. J. and Fried C. M, "Comparison and compressive and tensile properties of Mg based MMCs”, J. Mater. Sci. Technol., January 1993, 9, 35-41. 